\title{
COVID-19 in Pregnancy and the Pandemia Process in Gynecology and Obstetrics Clinic
}

\author{
(D) Veli Mihmanlı
}

University of Health Sciences Turkey, Prof. Dr. Cemil Taş̧̧ığlu City Hospital, Clinic of Gynecology and Obstetrics, İstanbul, Turkey

\section{Abstract}

The clinical symptoms of Coronavirus Disease-2019 (COVID-19) in pregnant women are similar to those of non-pregnant individuals. A positive test for Severe Acute Respiratory syndrome-coronavirus 2 usually confirms the diagnosis of COVID-19, but it should be known that there may be false positive and false negative results. Pregnancy does not increase susceptibility to infection and does not worsen the clinical course. The incidence of preterm delivery and cesarean delivery is high in infected pregnant women with pneumonia. It is recommended for use in standard indications in pregnant women with suspected or diagnosed COVID-19, since pregnant women between 24+0-33+6 weeks of gestation and with high risk of preterm delivery within seven days have proven benefits of antenatal betamethasone administration. Themode of delivery is determined by standard obstetric indications, including the mother's acute decompensation. There are advantages of preferringn euro axial anesthesia. Late clamping of the umbilical cord is not recommended. During the pandemic period, the necessary precautions were taken in line with there commendations of the Scientific Committees in the Gynecologyand Obstetrics Clinic, as in our entire hospital, and the pregnancy follow-up and birth processes of our mothers were tried to continue in a healthy manner.

Keywords: Pergnancy, COVID-19, pandemia

\section{INTRODUCTION}

Coronaviruses are a large family of viruses that can cause disease in animals or humans. In humans, it is known to cause common cold or more severe respiratory infections such as Middle East Respiratory syndrome and Severe Acute Respiratory syndrome (SARS). The most recently discovered coronavirus causes Coronavirus Disease-2019 (COVID-19). COVID-19 has become an pandemic that affects the whole world today (1).

\section{Prevention}

Pregnant women should also follow recommendations such as social distance, hand hygiene, wearing a mask in order not to be exposed to the virus. Women with an epidemiological history of contact should be monitored. Pregnant women with children should pay more attention. COVID-19 in children can usually be mild or asymptomatic. The possibility of transmission of the disease to pregnant women from asymptomatic or presymptomatic individuals in the incubation period should be considered (2-4).

\section{Clinical Features}

All pregnant women should be monitored in terms of the development of COVID-19 symptoms and signs, especially if they are in close contact with people who have been diagnosed as having COVID-19 or investigated for COVID-19. In a systematic review of 356 pregnant women, the most common symptoms were; fever (67\%), cough (66\%), shortness of breath (7\%), sore throat (7\%), fatigue (7\%), and myalgia (6\%). Rhinorrhea/nasal congestion, loss of appetite, nausea/vomiting, headache, and odor and/or taste abnormalities were also among the symptoms. Laboratory findings were; Iymphopenia (14\%), mild increase in liver enzymes (5\%), and thrombocytopenia (1\%). Clinical findings were similar to those who were not pregnant (5). According to 
the National Institute of Health, the disease was classified as asymptomatic or presymptomatic infection, mild, moderate, severe, and critical disease (6). In the study in which 147 pregnant women were included, $8 \%$ of the pregnant women were in the severe disease group and $1 \%$ were in the critical disease group (7). According to the available data; pregnancy and childbirth do not increase the risk of SARS-coronavirus 2 (CoV-2) infection and do not worsen the course of the disease compared to nonpregnant women of the same age. Most infected mothers (>90\%) recover before giving birth $(8,9)$.

\section{Pregnancy Complications}

The possibility of preterm birth and cesarean delivery increases. In a systematic review including 252 pregnant women with COVID-19, the birth rate before 37 weeks was reported as 15\% and the cesarean rate as $70 \%$. Hypoxemia caused by maternal fever and severe pneumonia may cause preterm labor, preterm membrane rupture and abnormal fetal heartbeat patterns. However, preterm birth can occur without serious respiratory disease. Another reason for the high rate of cesarean section may be the belief that maternal condition will improve by terminating the delivery by elective cesarean section in pregnant women with findings of severe respiratory disease. Although the data on first trimester are limited, the risk of spontaneous abortion does not appear to increase. Although there is anxiety that hyperthermia due to the disease may cause an increase in anomalies such as neural tube defect in pregnant women with COVID-19 in the period of organogenesis, theoretically, this situation has not been observed. More than $95 \%$ of newborns are in good condition. Complications related to the newborn are mostly related with preterm birth and adverse intrauterine environment resulting from critical maternal disease $(5,10-12)$.

Possible vertical transmission of peripartum maternal infection (transmission from mother to fetus) has been reported in the third trimester. Vertical transmission is possible but rare. Neonatal results are generally good unless there are problems such as preterm delivery or placental abruption. Data on fetal sequelae due to maternal infection in the first and second trimesters are insufficient. There are no accepted criteria for vertical transmission. Assessment of virus IgM level in cord blood and sampling of neonatal nasopharynx, amnion-chorion and placental tissue using aseptic technique immediately after birth have been recommended. Amniotic fluid obtained during cesarean section can also be tested. Vaginal and amniotic fluid samples have been found to be negative in women with positive test for SARS-CoV-2 in the nasopharynx. Viremia rates in patients with COVID-19 appear to be low (1\%) and transient, suggesting that vertical transmission will not be common. In the studies conducted, viruses were detected in a few patients, but no evidence of infection was found in the placentas. The extent and clinical significance of the vertical transmission remain unclear. A few patients with possible vertical contamination were reported based on the laboratory and/or clinical findings of the newborn (13-16).

\section{Diagnosis}

In patients with new onset of fever, chills and/or respiratory symptoms, (e.g. cough, shortness of breath) the possibility of COVID-19 should be considered. It should also be considered in patients with severe lower respiratory tract disease with no apparent cause. Being in or traveling to an area where there has been close contact with a confirmed or suspected patient with COVID-19 in the past 14 days should raise suspicion. In case of doubt, a nasopharyngeal swab sample should be taken and the SARS-CoV-2 RNA test should be performed with RT-PCR. It usually confirms the diagnosis of COVID-19, although a positive reverse transcription-polymerase chain reaction (RT-PCR) result may rarely be false positive. False negative tests were reported more frequently in the first day of symptoms and in the previous four days and in pregnant women. If the first nasopharyngeal test is negative but the suspicion of COVID-19 persists, the test should be repeated 24 hours later or within a few days. Infection control measures for COVID-19 should continue while reassessing. The next two negative samples usually rule out infection. In most patients hospitalized with a diagnosis of COVID-19, a chest $x$-ray is sufficient for the initial assessment of lung complications. A single chest $\mathrm{X}$-ray has a very low fetal radiation dose of 0.0005 to $0.01 \mathrm{mGy}$. Computed tomography (CT) can be used by taking the necessary precautions when necessary. Fetal radiation dose for a routine lung CT is also low and not associated with an increased risk of fetal anomalies or pregnancy loss. In pregnant women with COVID-19 with pneumonia, if pulmoner sonography is performed during obstetric ultrasonography, it can be helpful in making early diagnosis. Some laboratory abnormalities (hemolysis, elevated liver enzyme levels, thrombocytopenia) associated with COVID-19 in pregnant women are as in severe preeclampsia and HELLP syndrome. These diagnoses should be considered and it should be remembered that they may be found with COVID-19 (17-20).

\section{Antenatal Care}

Pregnant women with a history of epidemiological contact with a person with suspected or confirmed COVID-19 should be isolated and monitored for symptoms. The further 
evaluation and treatment of patients who become symptomatic depends on the severity of the disease, comorbidities and clinical condition. Patients with at least moderate illness are hospitalized. Professional organizations such as the American College of Obstetricians and Gynecologists (ACOG), The Society for Maternal-Fetal Medicine, and the Royal College of Obstetricians and Gynaecologists have published their recommendations on prenatal care during the COVID-19 pandemic including; restriction of visitors during examinations, wearing at least surgical masks by all patients and healthcare workers, reducing the number of prenatal visits especially in pregnant women without high risk pregnancy, taking measures to ensure that pregnant women, including high-risk pregnant women, stay less time in the office (75 g oral glucose tolerance test (OGTT) instead of two-stage $50 \mathrm{~g}$ OGTT), using the cell-free DNA test instead of combined test in screening for Down syndrome, duration and frequency of obstetric ultrasonography, biophysical profile, and Nonstress test (NST) (20-24).

\section{Medical Management of Pregnant Women with COVID-19}

Most pregnant women with confirmed or suspected COVID-19 are in a mild form of illness and do not need hospital care if there are no obstetric problems such as preterm birth. During home care, pregnant women should count fetal movements and report them in case of a decrease. Pregnant women with mild disease and comorbidity or with moderate/critical disease should be hospitalized in multidisciplinary $3^{\text {rd }}$ or $4^{\text {th }}$ level hospitals with obstetric and intensive care units (ICU). Fetal monitoring is an important issue in pregnant women during viability weeks. The frequency of fetal follow up depends on gestational age, stability of maternal vital findings, and maternal comorbidities. NST can be performed once or twice a day in patients with a stable general condition. In patients whose condition is not stable, continuous fetal monitorization is preferred, as emergency caesarean delivery may be required. An abnormal fetal heart trace can indicate the need for maternal oxygen therapy. Monitoring pregnant patients for signs and symptoms of preterm birth is a routine component of obstetric care (25).

The general supportive care of a patient with critical COVID-19 pneumonia is similar to that of patients with Acute Respiratory Distress syndrome (ARDS) for other reasons. Common complications of COVID-19-related ARDS include acute kidney damage, elevated liver enzymes, and heart damage (e.g. cardiomyopathy, pericarditis, pericardial effusion, arrhythmia, sudden cardiac death). During pregnancy, maternal peripheral oxygen saturation $\left(\mathrm{SpO}_{2}\right)$ should be kept $>95 \%$. If the $\mathrm{SpO}_{2}$ drops below 95\%, an arterial blood gas is taken to measure the partial oxygene pressure $\left(\mathrm{PaO}_{2}\right)$. Maternal $\mathrm{PaO}_{2}$ greater than $70 \mathrm{mmHg}$ is desirable to maintain a proper oxygen diffusion gradient from the maternal side of the placenta to the fetal side. In the ICU, severely ill patients with COVID-19 are generally recommended to be placed in the prone position. Some ICUs have applied this approach to pregnant women, but even the semi-prone position in the later weeks of pregnancy can be difficult for pregnant women. Permissive hypercapnia $\left(\mathrm{PCO}_{2}<60 \mathrm{mmHg}\right)$ and extracorporeal membran oxygenation do not appear to be harmful to the fetus if indicated for ARDS management, but the data are limited $(26,27)$.

Direct data between COVID-19 and thromboembolic risk are limited, but suggest an increased risk. Routine pharmacological prophylaxis for venous thromboembolism (VTE) is recommended in all hospitalized patients with COVID-19 without contraindications (bleeding, severe thrombocytopenia). For antepartum prophylaxis, unfractionated heparin $5000 \mathrm{U}$ subcutaneously every 12 hours is a reasonable dose (28).

Several agents (Remdesivir, lopinavir-ritonavir, ribavirin, baricitinib) are being evaluated for COVID-19 treatment. Some observational studies suggest that the administration of hydroxychloroquine or chloroquine is generally not beneficial, has adverse maternal effects such as abnormal heart rhythms (ventricular tachycardia), especially when taken in conjunction with other QTC prolonging drugs. Therefore, these drugs should not be used for COVID-19 treatment outside of ongoing randomized trials. Hydroxychloroquine crosses the placenta. In animal studies, accumulation in fetal ocular tissue has been observed. However, no increase in fetal ocular abnormality has been observed in the treatment of pregnant women with malaria and systemic lupus erythematosus. However, the available data are limited and the risk to the fetus cannot be excluded when used at different doses for other indications. Many other drugs are used in research. One such drug is lopinavir-ritonavir, which is used primarily for the treatment of HIV infection also in pregnancy. It crosses the placenta and may increase the risk of preterm birth, but no increased teratogenic risk has been observed in humans. Investigational drugs for COVID-19, which are known to be teratogenic, include ribavirin and barisitinib (29).

\section{Drugs used in pregnancy complications}

Betamethasone: Since antenatal betamethasone administration has proven benefits in pregnant women with high preterm birth risk within seven days between $24+0-33+6$ weeks of gestation, ACOG recommended its use in standard indications in pregnant women with suspected or confirmed COVID-19 (25). 
Low-dose aspirin: The decision to continue the drug in pregnant women with suspected or confirmed COVID-19 should be personalized. For example, use for preeclampsia prophylaxis is probably not beneficial in pregnant women with severe or critical illness or near term (25).

Non-steroidal anti-inflammatory drugs: There is no clinical or population-based direct data. Therefore, World Health Organization (WHO) does not recommend avoiding these drugs in patients with COVID-19 in the presence of clinical indications (30).

Tocolysis: The tocolytic agent preferred in pregnant women with confirmed or suspected COVID-19 is nifedipine.

\section{Delivery Timing in Infected Women}

If pregnant women with mild COVID-19 do not have medical and obstetric problems, it is ideal to have the delivery on time after negative test results are obtained following isolation. Thus, the risk of postnatal infection of the newborn is minimized. In those with medical and obstetric (membranoplasty, preeclampsi, etc.) complications, the time of delivery is individualized according to the protocols available for the specific medical problem. In those with severe and critical illness, whether the mother's respiratory disease will recover with birth and the risk of postnatal transmission is uncertain when maternal symptoms are in the acute period. As a result of increased normal oxygen consumption and decreased functional residual capacity in pregnant women, the general condition may deteriorate rapidly in patients with pneumonia. In the third trimester, in the presence of multiple pregnancy or polyhydramnios, excessive uterine dysfunction may impair respiratory function. For some patients with COVID-19 with a pregnancy of $>32-34$ weeks who are hospitalized due to pneumonia but not intubated, it is recommended to perform delivery before deterioration of pulmonary condition and development of fetal distress. It is recommended postponing delivery due to high morbidity and mortality risk in pregnancies $<32$ weeks. It is difficult to decide on the timing of delivery in pregnant women intubated due to COVID-19. Although delivery is advocated in pregnant women $>32-34$ weeks if the patient's condition is stable, it should not be forgotten that it may worsen the mother's condition. In pregnant women $<32$ weeks, fetal monitoring is generally recommended for perinatal benefit as long as the maternal condition remains stable or improves $(21,25,31)$.

\section{Management of Delivery}

All patients admitting for delivery should be questioned and controlled for symptoms and signs of COVID-19 and suspected contact. Suspected pregnant women should be given priority in terms of testing. Two hundred and fifteen pregnant women who were admitted for delivery in New York were screened for COVID-19, and 33 (15\%) pregnant women tested positive. Four of these pregnant women were symptomatic and 29 pregnant women (13.5\%) were asymptomatic. Healthcare professionals should use their personal protective equipment when dealing with confirmed or suspected COVID-19 pregnant women. Strong exhalation, especially during active labor, may reduce the effectiveness of the mask the patient uses to prevent the spread of the virus. The follow-up and treatment of pregnant women with suspected or confirmed COVID-19 should be done in isolated rooms with negative pressure in the hospital and surgical masks should be worn during labor. In pregnant women with COVID-19, the type of delivery is determined according to the standard obstetric indications, including the acute decompensation state of the mother. Pregnant women who will undergo planned induction or cesarean section should be hospitalized the day before and be screened for COVID-19. In patients with confirmed or suspected COVID-19, neuraxial anesthesia is not contraindicated and has several advantages in patients giving birth such as it provides good analgesia and thus reduces cardiopulmonary stress caused by pain and anxiety. If general anesthesia is applied (intubation and extubation), special personal protective equipment (N-95 masks, etc.) should be used by all relevant healthcare providers during cesarean delivery due to the risk of aerosolization. The use of magnesium sulfate for maternal eclamptic seizure prophylaxis and/or neonatal neuroprotection in suspected or confirmed COVID-19 positive pregnant women should be decided in consultation with maternal-fetal medicine, respiratory diseases and ICUs, considering the risk of respiratory depression. Labor management usually does not change in pregnant women with asymptomatic or mild COVID-19. Personal contact in the maternity unit should be limited as much as possible. Balloon catheters can be used for cervical ripening when necessary. Induction can shorten labor time. Continuous fetal montorization is recommended during labor. In more severe patients with respiratory distress or pneumonia, fluid intake and output should be carefully monitored, and aggressive hydration should be avoided as pulmonary edema may worsen maternal oxygenation. Oxygen therapy for fetal resuscitation should be abandoned, as intrapartum oxygen has no proven fetal benefit. The nasal cannula and face mask used are in contact with the maternal respiratory tract and secretions, so the use of such equipment increases the risk of contamination between 
patients and healthcare providers. ACOG does not recommend delayed clamping of the umbilical cord because of the risk of transmission of pathogens from an infected mother to the fetus (32-35).

\section{Postpartum Care}

In postpartum women with COVID-19, VTE prophylaxis should be considered based on individual risk assessment. In the mild disease group, vital signs should be evaluated in the first 24 hours after normal birth and every 4 hours during the first 48 hours after cesarean. For those with moderate illness, monitoring with pulse oximetry is recommended for the first 24 hours or until symptoms resolve. Serious or critical illness requires very close maternal follow-up in the ICU. Babies of mothers with COVID-19 are considered at risk of COVID-19. The test is performed in them and they are isolated from other healthy babies and followed up by taking infection control measures. The risk of transmission of SARS-CoV-2 through breast milk is uncertain. More data are needed to assess the risk of viral transmission. There is a general consensus that breastfeeding should be encouraged, as it has many benefits for mother and baby. Since breast milk is a source of antibodies, it can protect the newborn from the virus like other anti-infective factors $(21,35,36)$.

\section{Pandemic Process in the Gynecology and Obstetrics Clinic}

The WHO defined the COVID-19 outbreak as an "international public health emergency" on January 30. WHO declared on March 11 that it was a pandemic due to the spread and severity of the coronavirus, which emerged in China, called SARS-CoV-2 and spread to many countries. The first patient with COVID-19 in our country was also detected on March 11. As in our entire hospital, necessary measures were taken in the gynecology and obstetrics clinic in line with the recommendations of the scientific committees. Antenatal follow-up of all pregnant women including mother candidates with suspected or confirmed COVID-19 was performed. Routine outpatient clinic services continued with reducing the number of outpatient clinic rooms. Elective surgeries were postponed, except for emergent ones. Endoscopic operations were not performed due to the risk of aerosolization. In order to provide birth and care services to patients with suspected or confirmed COVID-19 without any complications, especially in terms of preventing disruption in antenatal examinations of pregnant women; we have worked in coordination with the Infectious diseases clinic, pediatric health and diseases clinic, neonatal unit and anesthesiology-intensive care clinic. A separate outpatient clinic with ultrasonography and NST device and a separate ward for antenatal and postpartum patients in which the health care was provided by physicians, midwives and nurses with personal protective equipment provided and where visitors were not allowed, were organized. A separate delivery room and cesarean operating room have been created for patients with suspected or confirmed COVID-19. After normal delivery or cesarean delivery, the baby is taken to the newborn ICU with a transport incubator and is isolated from other babies and followed up. On March 31, 2020, a symptomatic pregnant woman was diagnosed as having COVID-19 by the infectious diseases clinic in our hospital. The patient was hospitalized and treatment was started. In the following days, the follow-up and treatment of the pregnant women with suspected or confirmed COVID-19 have been made in the ward allocated to them. A specialist resident has been appointed by the hospital senior management for this ward every day. The transfer of the patients scheduled for a cesarean section to the operating room and surgeries have been carried out by taking appropriate precautions (Algorithm 1). Every pregnant who has been admitted to the outpatient clinic and emergency delivery clinic has been evaluated for COVID-19 (Figure 1). Suspected patients have been consulted with the infectious diseases clinic and related clinics according to their symptoms/ findings. Pregnant women who have been admitted directly to the infectious diseases clinic through the emergency unit and hospitalized, have been treated by hospitalization in the ward prepared for the pregnant women with suspected or confirmed COVID-19 and their obstetric follow-up has been made. All patients hospitalized in this ward have been evaluated daily by infectious diseases and, when necessary, chest diseases and internal medicine specialists, and their treatments have been arranged. Patients whose general condition has deteriorated, who have had dyspnea and increased respiratory rate and whose oxygen saturation could not achieve desired values despite nasal oxygen support have been evaluated by intensive care specialists. The treatment of patients in need of intensive care has been continued in the ICU.

\section{CONCLUSION}

Clinical signs of COVID-19 in pregnant women are similar to clinical signs of non-pregnant individuals. A positive test for SARS-CoV-2 usually confirms the diagnosis of COVID-19, but it should be noted that there may be false positive and false negative results. Pregnancy does not increase susceptibility to infection and does not worsen the clinical course. The incidence of preterm birth and cesarean delivery is high in infected pregnant women with pneumonia. 


\section{Symptoms are evaluated}

- Fever $\geq 38^{\circ}$ and/or

- Dry cough-shortness of breath

- Throat ache

- Weakness-Fatigue-Muscle pain

- Diarrhea

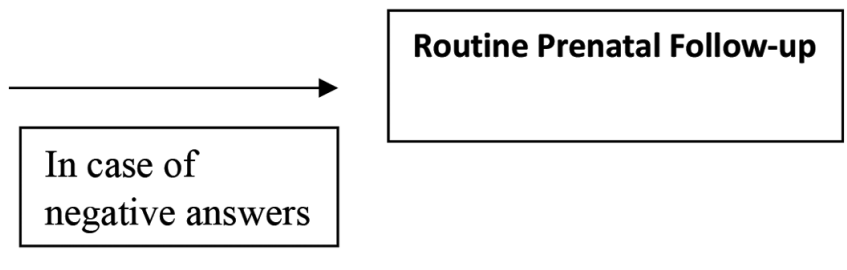

In case of a positive answer

The severity of the disease is evaluated

- Respiratory distress

- A feeling of pain or pressure in the chest

- Vomiting

- Vertigo

- Impaired consciousness
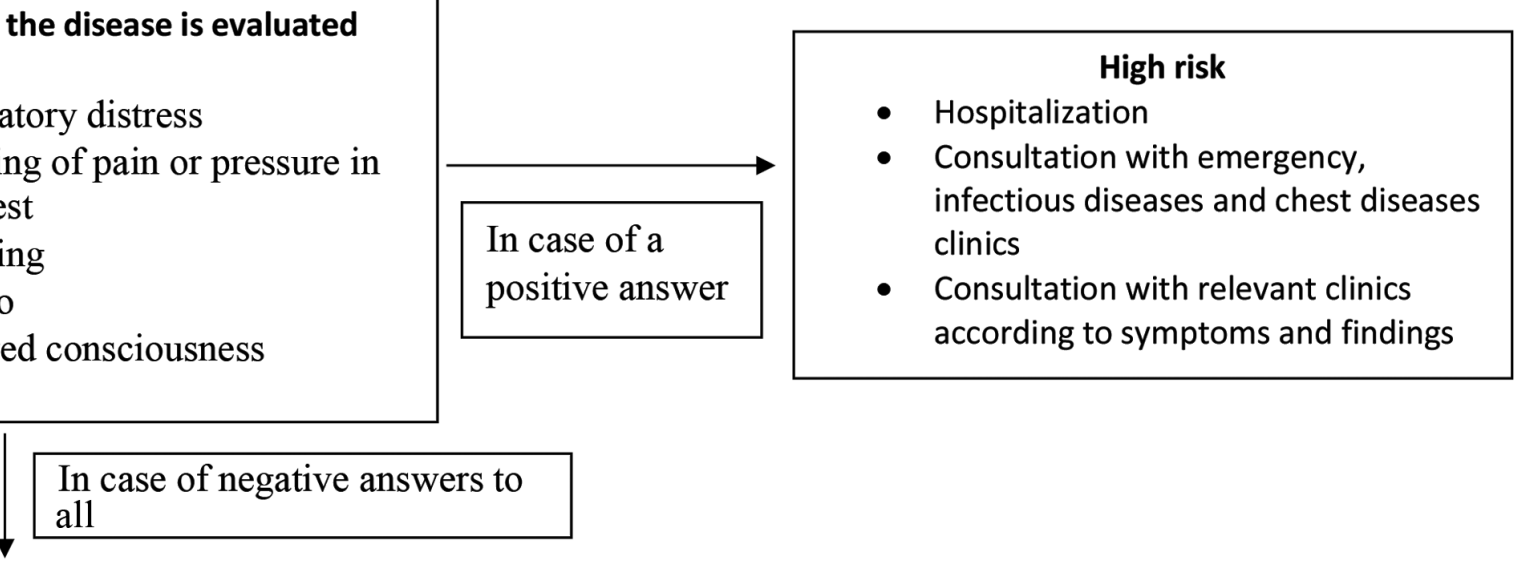

\section{Clinical risks are evaluated}

- Comorbidities

(hypertension, diabetes, asthma, HIV, chronic heart disease, chronic kidney disease)

- Obstetric problems (preterm labor, etc.)

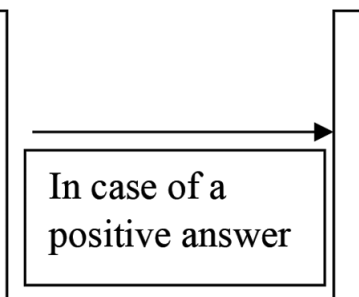

Moderate risk

- Hospitalization

- Consultation with infectious diseases and chest diseases clinics and consultation with relevant clinics according to symptoms and findings

\section{In case of negative answers to all}

Low risk

- Consultation with infectious diseases clinic

- Follow up 


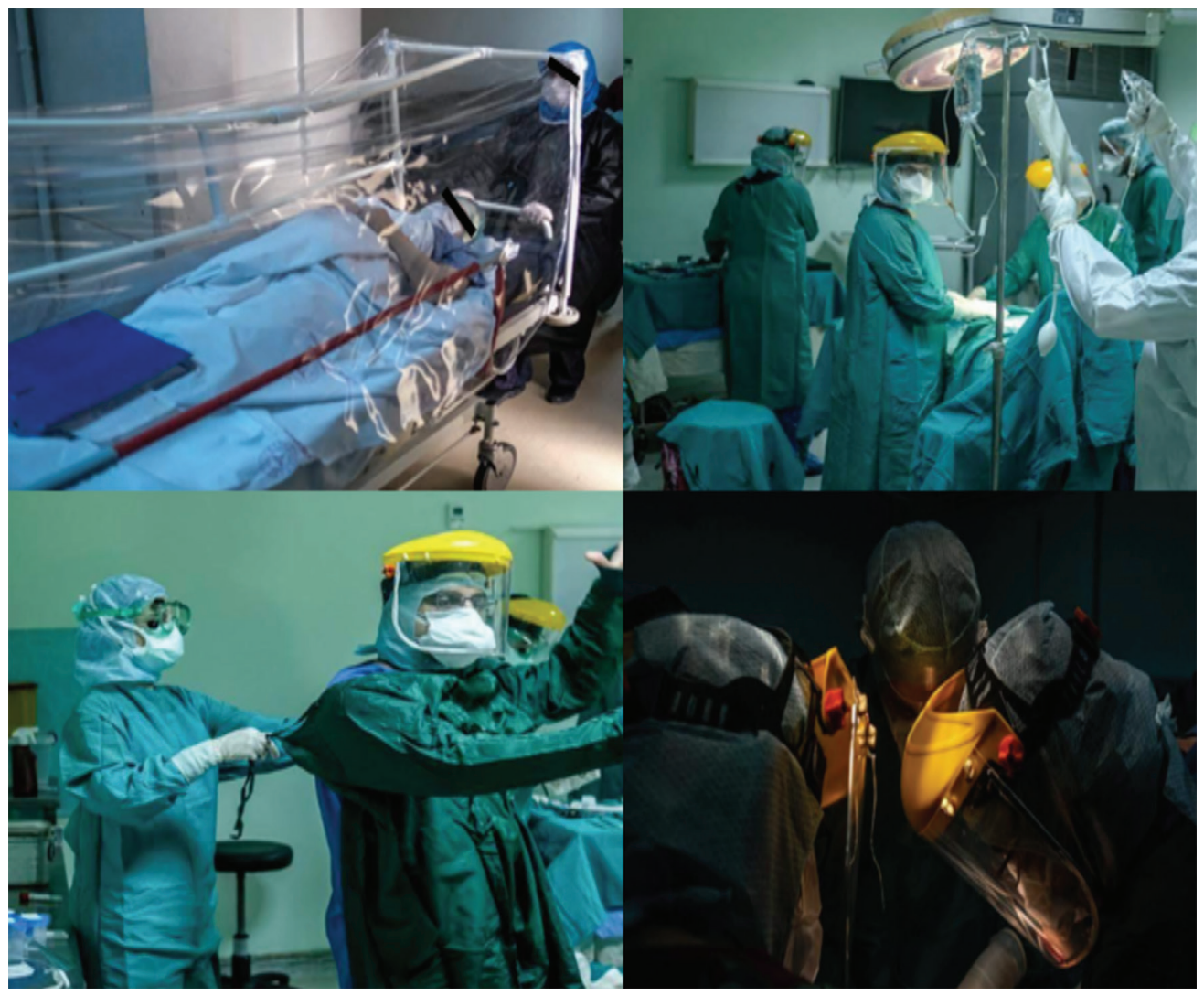

Figure 1. Transfer of a pregnant woman with COVID-19 to the operating room and cesarean operation

COVID-19: Coronavirus Disease-2019

\section{Ethics}

Peer-review: Externally and internally peer-reviewed.

Financial Disclosure: The author declared that this study received no financial support.

\section{REFERENCES}

1. Coronavirus disease (COVID-19) pandemic. Available from: URL: https:// www.who.int/emergencies/diseases/novel-coronavirus-2019

2. Public Health Guidance for Community-Related Exposure. Available from: URL: https://www.cdc.gov/coronavirus/2019-ncov/php/publichealth-recommendations.html

3. Yu P, Zhu J, Zhang Z, Han Y. A Familial cluster of infection associated with the 2019 novel coronavirus indicating possible person-to-person transmission during the incubation period. J Infect Dis 2020;221:175761.

4. Bai Y, Yao L, Wei T, Tian F, Jin DY, Chen L, et al. Presumed asymptomatic carrier transmission of COVID-19. JAMA 2020;323:1406-7.

5. Elshafeey F, Magdi R, Hindi N, Elshebiny M, Farrag N, Mahdy S, et al. A systematic scoping review of COVID-19 during pregnancy and childbirth. Int J Gynaecol Obstet 2020;150:47-52

6. NIH COVID-19 Treatment Guidelines. Management of persons with COVID-19.Availablefrom:URL:https://www.covid19treatmentguidelines. nih.gov/overview/management-of-covid-19/
7. Report of the WHO-China joint mission on Coronavirus Disease 2019 (COVID-19). 16-24 February 2020. Available from: URL: https://www. who.int/docs/default-source/coronaviruse/who-china-joint-mission-oncovid-19-final-report.pdf

8. Wu Z, McGoogan JM. Characteristics of and important lessons from the Coronavirus Disease 2019 (COVID-19) Outbreak in China: summary of a report of 72314 cases from the Chinese Center for Disease control and prevention. JAMA 2020;323:1239-42.

9. Breslin N, Baptiste C, Gyamfi-Bannerman C, Miller R, Martinez R, Bernstein K, et al. COVID-19 infection among asymptomatic and symptomatic pregnant women: two weeks of confirmed presentations to an affiliated pair of New York City hospitals. Am J Obstet Gynecol MFM 2020;2:100118.

10. Juan J, Gil MM, Rong Z, Zhang Y, Yang H, Poon LC. Effects of coronavirus disease 2019 (COVID-19) on maternal, perinatal and neonatal outcomes: a systematic review. Ultrasound Obstet Gynecol 2020;56:15-27.

11. Li J, Wang Y, Zeng Y, Song T, Pan X, Jia M, et al. Critically ill pregnant patient with COVID-19 and neonatal death within two hours of birth. Int J Gynaecol Obstet 2020;150:126-8.

12. Edwards MJ. Review: hyperthermia and fever during pregnancy. Birth Defects Res A Clin Mol Teratol 2006;76:507-16.

13. Qiu L, Liu X, Xiao M, Xie J, Cao W, Liu Z, et al. SARS-CoV-2 is not detectable in the vaginal fluid of women with severe COVID-19 infection. Clin Infect Dis 2020:ciaa375. 
14. Chen H, Guo J, Wang C, Luo F, Yu X, Zhang W, et al. Clinical characteristics and intrauterine vertical transmission potential of COVID-19 infection in nine pregnant women: a retrospective review of medical records. Lancet 2020;395:809-15.

15. Wang W, Xu Y, Gao R, Lu R, Han K, Wu G, et al. Detection of SARS-CoV-2 in different types of clinical specimens. JAMA 2020;323:1843-4.

16. Baud D, Greub G, Favre G, Gengler C, Jaton K, Dubruc E, et al. Secondtrimester miscarriage in a pregnant woman with SARS-CoV-2 infection. JAMA 2020;323:2198-200.

17. Sethuraman N, Jeremiah SS, Ryo A. Interpreting diagnostic tests for SARS-CoV-2. JAMA 2020;323:2249-51.

18. Kelly JC, Dombrowksi M, O'neil-Callahan M, Kernberg AS, Frolova AI, Stout MJ. False-negative testing for severe acute respiratory syndrome coronavirus 2: consideration in obstetrical care. Am J Obstet Gynecol MFM 2020;2:100130.

19. Moro F, Buonsenso D, Moruzzi MC, Inchingolo R, Smargiassi A, Demi $L$, et al. How to perform lung ultrasound in pregnant women with suspected COVID-19. Ultrasound Obstet Gynecol 2020;55:593-8.

20. Buonsenso D, Raffaelli F, Tamburrini E, Biasucci DG, Salvi S, Smargiassi $A$, et al. Clinical role of lung ultrasound for the diagnosis and monitoring of COVID-19 pneumonia in pregnant women. Ultrasound Obstet Gynecol 2020;56:106-9.

21. https://www.acog.org/clinical/clinical-guidance/practice-advisory/ articles /2020/03/ novel-coronavirus-2019

22. SMFM. Coronavirus (COVID-19). Available from: URL: https://www.smfm. org/covid19

23. RCOG. Coronavirus (COVID-19) infection and pregnancy. Available from: URL: https://www.rcog.org.uk/coronavirus-pregnancy

24. Berthelot N, Lemieux R, Garon-Bissonnette J, Drouin-Maziade C, Martel E, Maziade M. Uptrend in distress and psychiatric symptomatology in pregnant women during the coronavirus disease 2019 pandemic. Acta Obstet Gynecol Scand 2020;99:848-55.

25. ACOG. COVID-19 FAQs for obstetrician-gynecologists, obstetrics Available from: URL: https://www.acog.org/clinical-information/physician-faqs/ covid-19-faqs-for-ob-gyns-obstetrics

26. Anesi GL, Manaker S, Finlay G, Bloom A. Coronavirusdisease 2019 (COVID-19): Critical care and airway management issues. Available from: URL: https://www.uptodate.com/contents/coronavirus-disease2019-covid-19-critical-care-and-airway-management-issues

27. Webster CM, Smith KA, Manuck TA. Extracorporeal membrane oxygenation in pregnant and postpartum women: a ten-year case series. Am J Obstet Gynecol MFM 2020;2:100108.

28. American Society of Hematology. COVID-19 and VTE/anticoagulation: frequently asked questions. Available from: URL: https://www. hematology.org/covid-19/covid-19-and-vte-anticoagulation

29. Kim AY, Gandhi RT, Hirsch MS, BloomA. Coronavirus disease 2019 (COVID-19): Management in hospitalized adults. Available from: URL: https://www.uptodate.com/contents/coronavirus-disease-2019-covid19-management-in-hospitalized-adults

30. ScienceAlert. Updated: WHO now doesn't recommend avoiding ibuprofen for COVID-19 symptoms. Available from: URL: https://www. sciencealert.com/who-recommends-to-avoid-taking-ibuprofen-forcovid-19-symptoms

31. Stephens AJ, Barton JR, Bentum NA, Blackwell SC, Sibai BM. General guidelines in the management of an obstetrical patient on the laborand delivery unitduring the COVID-19 pandemic. Am J Perinatol 2020;37:829-36.

32. CDC. Considerations for inpatient obstetric healthcare settings. CDC interim guidance on infection prevention and control for COVID-19. Available from: URL: https://www.cdc.gov/coronavirus/2019-ncov/hcp/ inpatient-obstetric-healthcare-guidance.html

33. Sutton D, Fuchs K, D'Alton M, Goffman D. Universal screening for SARSCoV-2 in women admitted for delivery. N Engl J Med 2020;382:2163-4.

34. Jamieson DJ, Steinberg JP, Martinello RA, Perl TM, Rasmussen SA. Obstetricians on the Coronavirus Disease 2019 (COVID-19) front lines and the confusing world of personal protective equipment. Obstet Gynecol 2020;135:1257-63.

35. Boelig RC, Manuck T, Oliver EA, Di Mascio D, Saccone G, Bellussi F, et al. Labor and delivery guidance for COVID-19. Am J Obstet Gynecol MFM 2020;2:100110

36. Meek JY, Abrams SA, Hoppin AG. Infant benefits of breast feding Available from: URL: https://www.uptodate.com/contents/infantbenefits-of-breastfeeding 